\title{
In-Vitro Regeneration of Citrus sinensis (L.) Osbeck from Mature Seed Derived Embryogenic Callus on Different Solid Basal Media
}

\author{
Md. Nazmul Hasan, Mohammed Raqibul Hasan, Shakhawat Hossain Foysal, Hammadul Hoque, \\ Md. Fahim Khan, Md. Fahmid Hossain Bhuiyan, Shamsul H. Prodhan*
}

Department of Genetic Engineering and Biotechnology, Shahjalal University of Science and Technology, Sylhet, Bangladesh

Email: *shamsulhp@yahoo.com

How to cite this paper: Hasan, M.N., Hasan, M.R., Foysal, S.H., Hoque, H., Khan, M.F., Bhuiyan, M.F.H. and Prodhan, S.H. (2019) In-Vitro Regeneration of Citrus sinensis (L.) Osbeck from Mature Seed Derived Embryogenic Callus on Different Solid Basal Media. American Journal of Plant Sciences, 10, 285-297.

https://doi.org/10.4236/ajps.2019.102022

Received: January 6, 2019

Accepted: February 23, 2019

Published: February 26, 2019

Copyright $\odot 2019$ by author(s) and Scientific Research Publishing Inc. This work is licensed under the Creative Commons Attribution-NonCommercial International License (CC BY-NC 4.0). http://creativecommons.org/licenses/by-nc/4.0/ (c) (i) \& Open Access

\begin{abstract}
In-vitro callus induction and regeneration method was developed using different plant growth regulators (PGRs), and basal media (Murashige and Skoog (MS), CHU (N6) and Gamborg (B5) media) of Citrus sinensis (L.) Osbeck. Observations of the effect of PGRs were carried out using different concentrations of 2,4-dichlorophenoxyacetic acid (2,4-D),1-naphthalene acetic acid (NAA) and combinations of 2,4-D and NAA using different basal media. This study found Citrus sinensis (L.) Osbeck exhibited a high frequency of callus induction on MS medium supplemented with $3 \mathrm{mg} / \mathrm{L}$ 2,4-D and callus induction frequency was $86.7 \% \pm 3.4 \%$ whereas N6 and B5 showed lower callus induction frequency of $83.3 \% \pm 8.8 \%$ and $82.2 \% \pm 1.9 \%$ respectively compared to that of MS media with supplementation of the same hormone. Among the induced calli, the morphological analysis showed only $40 \%$ - 50\% was embryogenic calli. Regeneration of plantlets from calli was done using different concentrations and combinations of auxin and cytokinin. The study showed that $3 \mathrm{mg} / \mathrm{L}$ 6-benzylaminopurine (BAP) supplemented medium has the maximum potential to promote regeneration of Citrus sinensis (L.) Osbeck from embryogenic calli with the frequency of $89.3 \% \pm 8.8 \%$ but no regeneration occurred from the non-embryogenic calli. The regenerated plantlets were rooted on MS medium with supplementation of $5 \mathrm{mg} / \mathrm{l} \mathrm{NAA}$. These observations in Citrus sinensis (L.) Osbeck regeneration will be helpful for genetic improvement with desired traits.
\end{abstract}

\section{Keywords}

Citrus sinensis (L.) Osbeck, PGRs, Basal Media, Regeneration 


\section{Introduction}

Citrus is a large genus that includes many cultivated species, (e.g. Citrus sinensis, Citrus reticulata, Citrus limon, Citrus grandis and Citrus paradise [1]). Citrus sinensis, is commonly known as orange or sweet orange that originated in southern China, where it has been cultivated for millennia. Oranges are now grown commercially worldwide in tropical, semi-tropical, and some warm temperate regions, and have become the most widely planted fruit tree in the world. Oranges are the world's most popular fruit and are eaten fresh and used for juice. In 2016, the global harvest area of orange was 3.97 million hectares and production was 73.19 million tons (FAO Stat, 2017). Exporters of orange in the world have exported 4.65 billion USD in 2016. Sweet orange accounted for approximately $60 \%$ of citrus production for both fresh fruit and processed juice consumption. Moreover, citrus fruits and juice are the prime sources of vitamin C, an important component of human nutrition [1]. Citrus fruits also have some unique botanical features, such as nucellar embryony (nucellus cells can develop into apomictic embryos that are genetically identical to mother plant). Consequently, somatic embryos grow much more vigorously than the zygotic embryos in seeds such that seedlings are essentially clones of the maternal parent [2]. Orange trees and fruits are susceptible to various pests and pathogens, including the Mediterranean fruit fly (Ceratitis capitata), numerous fungal leaf spots, blights, and root rots (including Cercospora, Colletotrichum, Fusarium, Phytophthora, and many others) and viruses that can significantly reduce yields. Production of Citrus sinensis (L.) Osbeck has been imperiled due to the introduction of several biotic (bacterial disease Citrus canker) and abiotic stress (cold, drought). No significant improvements have been made to combat these stresses by plant breeding [3]. Improvement of Citrus spp. by breeding methods is impeded by various aspects of citrus biology such as heterozygosity, nucellar polyembryony, sexual incompatibility and long juvenile period [4]. With the recent advances of plant biotechnology, it is possible to introduce exogenous genes in the plant genome, using gene transfer techniques. However, for efficient transgenic plant production, a previously defined tissue culture system for efficient plant regeneration, in association with a genetic transformation system for the gene introduction is necessary [5]. Accordingly, genetic transformation remains the main alternative in Citrus breeding programs such as development of Citrus tristeza virus (CTV) tolerant sour orange plants. The success of a genetic transformation program depends on the availability of an in vitro protocol that permits highly efficient shoot regeneration [6]. Combination of conventional breeding, biotechnological approaches and in vitro tissue culture methods could be potential for developing new citrus varieties. Although conventional breeding methods are being applied to improve citrus varieties, progress is slow [7]. The first successful regeneration of citrus plantlets from mature inter-nodal stem segments was reported using sweet orange [8]. Other investigators reported shoot induction 
from mature Citrus tissues [9] [10] [11]. However, the lack of efficient tissue culture protocols is one of the main barriers to improvement through breeding and biotechnological studies [12]. In this study, we aimed to establish a regeneration protocol of sweet orange (Citrus sinensis (L.) Osbeck.), which is the most important sweet orange variety cultivated globally and grown primarily for orange juice production.

\section{Materials and Methods}

Mature seeds of Citrus sinensis (L.) Osbeck was collected from Citrus research station, Jointa, Sylhet. Healthy and good quality seeds were used as explants in this study. First, the seeds were dehusked and washed several times with sterile distilled water. Then the explants were sterilized using $75 \%$ ethanol for $5 \mathrm{mi}$ nutes and followed by washing with sterile distilled water for several times. The explants were further sterilized with $0.1 \% \mathrm{HgCl}_{2}$ and Tween 20 . Finally, the explants were dried on sterilized filter paper after washing several times with sterile distilled water [13]. The sterilized explants were inoculated in test tubes containing MS (Merck), Chu N6 (Duchefa Biochemie) and Gamborg B5 (Duchefa Biochemie) media assigned as MSCIM, N6CIM and B5CIM respectively (Table 1) which were supplemented with different concentrations of 2,4-D and combinations of 2,4-D and NAA to induce callus (Tables 2-4). Media were prepared according to manufacturer protocol with the addition of $3 \%$ sucrose and the $\mathrm{pH}$ of the media was adjusted to 5.8 and then $0.7 \%$ agar was added in the media. Inoculated explants were incubated in a culture room under 16 hours of light (2000 lux) and 8 hours of dark, $25^{\circ} \mathrm{C} \pm 1^{\circ} \mathrm{C}$ temperature [14]. The explants were sub-cultured in an interval of $15 \pm 1$ days. Upon induction of calli, they were divided into two categories e.g. embryogenic and non-embryogenic through morphological evaluation. The calluses were then transferred to shoot induction media and incubated in a culture room under 16 hours photoperiods and 8 hours darkness, $25^{\circ} \mathrm{C} \pm 1{ }^{\circ} \mathrm{C}$ temperature, 2000 lux intensity of light conditions [15]. Shoot induction media was prepared using MS, chu N6, and B5 (MSSIM, N6SIM and B5SIM respectively) (Table 1) individually supplemented with different growth hormones and regulators (Tables 5-7). Regenerated plantlets were transferred to root inducing media under same culture room conditions. Root inducing media were prepared using MS, Chu N6 and Gamborg B5 (MSRtM, N6RtM and B5RtM respectively) containing 3\% sucrose with supplementation of $5 \mathrm{mg} / \mathrm{l} \mathrm{NAA}$ (Table 8). All the media used in this experiment were autoclaved at $121^{\circ} \mathrm{C}$ temperature, 15 psi pressures for 20 minutes. After root formation, the plantlets were acclimatized in pots containing garden soil mixed with biofertilizer in 1:1 ratio.

The frequency of callus induction, regeneration and rooting of Citrus sinensis (L.) Osbeck were calculated for three replications. Arithmetic mean (A.M.) and standard deviation (S.D.) were evaluated by analyzing data with Microsoft Excel 2007. 
Table 1. List of different media used in this experiment.

\begin{tabular}{ccccc}
\hline SL No & Description & Composition & Abbreviation \\
\hline 01 & Callus induction media I & MS salts and vitamins, 3\% sucrose, 0.7\%, agar, pH 5.8 and hormones & MSCIM \\
02 & Callus induction media II & N6 salts and vitamins, 3\% sucrose, 0.7\%, agar, pH 5.8 and hormones & N6CIM \\
03 & Callus induction media III & B5 salts and vitamins, 3\% sucrose, 0.7\%, agar, pH 5.8 and hormones & B5CIM \\
04 & Regeneration media I & MS salts and vitamins, 3\% sucrose, 0.7\%, agar, pH 5.8 and hormones & MSSIM \\
05 & Regeneration media II & N6 salts and vitamins, 3\% sucrose, 0.7\%, agar, pH 5.8 and hormones & N6SIM \\
06 & Regeneration media III & B5 salts and vitamins, 3\% sucrose, 0.7\%, agar, pH 5.8 and hormones & B5SIM \\
07 & Rooting Media I & MS salts and vitamins, 3\% sucrose, 0.7\%, agar, pH 5.8 & MSRtM \\
08 & Rooting Media II & N6 salts and vitamins, 3\% sucrose, 0.7\%, agar, pH 5.8 & N6RtM \\
09 & Rooting Media III & B5 salts and vitamins, 3\% sucrose, 0.7\%, agar, pH 5.8 & B5RtM \\
\hline
\end{tabular}

Table 2. The effects of 2,4-D, NAA and their combinations on explants of Citrus sinensis (L.) Osbeck, cultured on MSCIM for callus induction.

\begin{tabular}{|c|c|c|c|c|c|}
\hline \multicolumn{2}{|c|}{ MS + Hormone (mg/l) } & \multirow[b]{2}{*}{ Observations } & \multirow{2}{*}{$\begin{array}{l}\text { Callus \% } \\
(\mathrm{AM}) \pm \mathrm{SD}\end{array}$} & \multicolumn{2}{|c|}{ Organogenesis } \\
\hline 2,4-D & NAA & & & $\begin{array}{c}\text { Shoot (AM) } \\
\% \pm S D\end{array}$ & $\begin{array}{c}\text { Root (AM) } \\
\% \pm S D\end{array}$ \\
\hline 0 & 0 & No callus formation observed after 30 days & 0 & $48.9 \pm 10.2$ & $28.9 \pm 6.9$ \\
\hline 0.5 & 0 & No callus formation observed after 30 days & 0 & $40.0 \pm 12.0$ & $35.6 \pm 5.1$ \\
\hline 1.0 & 0 & Whitish compact non embryogenic callus observed after 25 days & $14.4 \pm 1.9$ & NR & NR \\
\hline 1.5 & 0 & Whitish compact non embryogenic callus observed after 25 days & $35.6 \pm 6.9$ & NR & NR \\
\hline 2.0 & 0 & Light greenish nodular embryogenic callus observed after 20 days & $64.5 \pm 6.9$ & NR & NR \\
\hline 2.5 & 0 & Light greenish nodular embryogenic callus ( $\sim 40 \%)$ observed after 25 days & $74.4 \pm 5.1$ & NR & NR \\
\hline 3.0 & 0 & Greenish nodular embryogenic callus ( $\sim 75 \%)$ observed after 25 days & $86.7 \pm 3.4$ & NR & NR \\
\hline 3.5 & 0 & Greenish nodular embryogenic callus ( $\sim 50 \%)$ observed after 25 days & $82.2 \pm 5.1$ & NR & NR \\
\hline 4.0 & 0 & Whitish compact non callus observed after 20 days & $66.7 \pm 3.4$ & NR & NR \\
\hline 4.5 & 0 & Whitish compact non embryogenic callus observed after 20 days & $54.5 \pm 3.9$ & NR & NR \\
\hline 5.0 & 0 & Whitish compact non embryogenic callus observed after 20 days & $51.1 \pm 5.1$ & NR & NR \\
\hline 0 & 1.0 & No response after 30 days & 0 & $58.9 \pm 8.4$ & $36.7 \pm 8.8$ \\
\hline 0 & 2.0 & Light yellowish compact callus found after 20 days & $33.3 \pm 10.0$ & $45.5 \pm 6.9$ & NR \\
\hline 0 & 3.0 & Light yellowish compact callus found after 20 days & $61.1 \pm 10.1$ & NR & NR \\
\hline 0 & 4.0 & Light yellowish compact callus found after 20 days & $52.2 \pm 6.9$ & NR & NR \\
\hline 2.0 & 0.5 & Light greenish compact callus found after 25 days & $66.7 \pm 6.7$ & NR & NR \\
\hline 2.0 & 1.0 & Light greenish compact callus found after 25 days & $73.33 \pm 8.8$ & NR & NR \\
\hline 2.0 & 1.5 & Greenish nodular embryogenic callus ( $40 \%)$ found after 25 days & $80.0 \pm 3.3$ & NR & NR \\
\hline 3.0 & 0.5 & Greenish nodular embryogenic callus ( $\sim 40 \%)$ found after 25 days & $76.7 \pm 6.7$ & NR & NR \\
\hline 3.0 & 1.0 & Yellowish compact callus found after 25 days & $57.8 \pm 11.7$ & NR & NR \\
\hline 3.0 & 1.5 & Yellowish compact callus found after 25 days & $52.2 \pm 6.9$ & NR & NR \\
\hline
\end{tabular}

* $\mathrm{AM}=$ Arithmetic Mean; $\mathrm{SD}=$ Standard Deviation; $\mathrm{NR}=$ No Response. 
Table 3. The effects of 2,4-D, NAA and their combinations on explants of Citrus sinensis (L.) Osbeck, cultured on N6CIM for callus induction.

\begin{tabular}{|c|c|c|c|c|c|}
\hline \multicolumn{2}{|c|}{$\mathrm{N} 6+$ Hormone $(\mathrm{mg} / \mathrm{l})$} & \multirow[b]{2}{*}{ Observations } & \multirow{2}{*}{$\begin{array}{c}\text { Callus \% (AM) } \\
\quad \pm \mathrm{SD}\end{array}$} & \multicolumn{2}{|c|}{ Organogenesis } \\
\hline $2,4-\mathrm{D}$ & NAA & & & $\begin{array}{c}\text { Shoot (AM) } \\
\% \pm \text { SD }\end{array}$ & $\begin{array}{l}\text { Root (AM) } \\
\% \pm \text { SD }\end{array}$ \\
\hline 0 & 0 & $\begin{array}{l}\text { No callus formation but germination were observed } \\
\text { after } 30 \text { days }\end{array}$ & $0.0 \pm 0.0$ & $25.6 \pm 8.2$ & $26.7 \pm 8.8$ \\
\hline 0.5 & 0 & $\begin{array}{l}\text { No callus formation but germination were observed } \\
\text { after } 30 \text { days }\end{array}$ & $0.0 \pm 0.0$ & $22.1 \pm 3.7$ & $31.1 \pm 5.1$ \\
\hline 1.0 & 0 & $\begin{array}{l}\text { No callus formation and no germination were observed } \\
\text { after } 30 \text { days }\end{array}$ & $0.0 \pm 0$ & $32.2 \pm 7.7$ & $26.7 \pm 6.7$ \\
\hline 1.5 & 0 & Whitish compacts callus found after 25 days & $35.6 \pm 6.9$ & NR & NR \\
\hline 2.0 & 0 & Greenish nodular callus found after 25 days & $67.8 \pm 1.9$ & NR & NR \\
\hline 2.5 & 0 & Greenish nodular callus found after 25 days & $83.3 \pm 8.8$ & NR & NR \\
\hline 3.0 & 0 & Greenish nodular callus found after 25 days & $82.2 \pm 5.1$ & NR & NR \\
\hline 3.5 & 0 & Yellowish compact callus found after 25 days & $75.6 \pm 6.9$ & NR & NR \\
\hline 4.0 & 0 & Yellowish compact callus found after 25 days & $68.9 \pm 1.9$ & NR & NR \\
\hline 4.5 & 0 & Yellowish compact callus found after 25 days & $62.2 \pm 9.6$ & NR & NR \\
\hline 5.0 & 0 & Yellowish compact callus found after 25 days & $60.0 \pm 8.8$ & NR & NR \\
\hline 0 & 1.0 & No callus formation observed after 30 days & $0.0 \pm 0.0$ & $41.1 \pm 8.4$ & $25.6 \pm 5.1$ \\
\hline 0 & 2.0 & No callus formation observed after 30 days & $0.0 \pm 0.0$ & $28.9 \pm 10.2$ & $22.2 \pm 6.9$ \\
\hline 0 & 3.0 & Whitish compacts callus found after 25 days & $58.9 \pm 7.7$ & NR & NR \\
\hline 0 & 4.0 & Whitish compacts callus found after 25 days & $61.1 \pm 5.1$ & NR & NR \\
\hline 2.0 & 0.5 & Greenish nodular callus found after 25 days & $62.2 \pm 3.9$ & NR & NR \\
\hline 2.0 & 1.0 & Greenish nodular callus found after 25 days & $76.7 \pm 3.3$ & NR & NR \\
\hline 2.0 & 1.5 & Greenish nodular callus found after 25 days & $77.8 \pm 6.9$ & NR & NR \\
\hline 3.0 & 0.5 & Greenish nodular callus found after 25 days & $75.6 \pm 5.1$ & NR & NR \\
\hline 3.0 & 1.0 & Yellowish compact callus found after 25 days & $54.5 \pm 6.9$ & NR & NR \\
\hline 3.0 & 1.5 & Yellowish compact callus found after 25 days & $51.1 \pm 5.1$ & NR & NR \\
\hline
\end{tabular}

${ }^{\star} \mathrm{AM}=$ Arithmetic Mean; SD = Standard Deviation; NR = No Response.

Table 4. The effects of 2,4-D, NAA and their combinations on explants of Citrus sinensis (L.) Osbeck, cultured on B5CIM for callus induction.

\begin{tabular}{|c|c|c|c|c|c|}
\hline \multicolumn{2}{|c|}{ B5 + Hormone $(\mathrm{mg} / \mathrm{l})$} & \multirow[b]{2}{*}{ Observations } & \multirow{2}{*}{$\begin{array}{l}\text { Callus \% } \\
(\mathrm{AM}) \pm \mathrm{SD}\end{array}$} & \multicolumn{2}{|c|}{ Organogenesis } \\
\hline 2,4-D & NAA & & & $\begin{array}{c}\text { Shoot (AM) } \\
\% \pm S D\end{array}$ & $\begin{array}{l}\text { Root }(\mathrm{AM}) \\
\% \pm \mathrm{SD}\end{array}$ \\
\hline 0 & 0 & $\begin{array}{l}\text { No callus formed and germination were observed } \\
\text { after } 30 \text { days }\end{array}$ & $0.0 \pm 0.0$ & $31.7 \pm 7.6$ & $48.7 \pm 8.0$ \\
\hline 0.5 & 0 & $\begin{array}{l}\text { No callus formed and germination were observed } \\
\text { after } 30 \text { days }\end{array}$ & $0.0 \pm 0.0$ & $28.3 \pm 10.4$ & $35.3 \pm 5.0$ \\
\hline
\end{tabular}




\section{Continued}

\begin{tabular}{|c|c|c|c|c|c|}
\hline 1.0 & 0 & $\begin{array}{l}\text { No callus formation and germination were observed } \\
\text { after } 30 \text { days }\end{array}$ & $0.0 \pm 0.0$ & $27 \pm 8.1$ & $42.0 \pm 7.2$ \\
\hline 1.5 & 0 & No callus formation observed after 30 days & $0.0 \pm 0.0$ & NR & NR \\
\hline 2.0 & 0 & Whitish compacts callus found after 25 days & $54.4 \pm 6.9$ & NR & NR \\
\hline 2.5 & 0 & Whitish compacts callus found after 25 days & $73.3 \pm 5.8$ & NR & NR \\
\hline 3.0 & 0 & Greenish nodular callus found after 25 days & $81.1 \pm 3.9$ & NR & NR \\
\hline 3.5 & 0 & Greenish nodular callus found after 25 days & $82.2 \pm 1.9$ & NR & NR \\
\hline 4.0 & 0 & Yellowish compact callus found after 25 days & $68.9 \pm 1.9$ & NR & NR \\
\hline 4.5 & 0 & Yellowish compact callus found after 25 days & $57.8 \pm 1.9$ & NR & NR \\
\hline 5.0 & 0 & Yellowish compact callus found after 25 days & $52.2 \pm 3.6$ & NR & NR \\
\hline 0 & 1.0 & No callus formation observed after 30 days & $0.0 \pm 0.0$ & NR & NR \\
\hline 0 & 2.0 & No callus formation observed after 30 days & $0.0 \pm 0.0$ & NR & NR \\
\hline 0 & 3.0 & Whitish compacts callus found after 25 days & $60.0 \pm 8.8$ & NR & NR \\
\hline 0 & 4.0 & Whitish compacts callus found after 25 days & $55.6 \pm 7.7$ & NR & NR \\
\hline 2.0 & 0.5 & Whitish compacts callus found after 25 days & $68.9 \pm 3.6$ & NR & NR \\
\hline 2.0 & 1.0 & Greenish nodular callus found after 25 days & $75.6 \pm 5.1$ & NR & NR \\
\hline 2.0 & 1.5 & Greenish nodular callus found after 25 days & $78.9 \pm 1.9$ & NR & NR \\
\hline 3.0 & 0.5 & Greenish nodular callus found after 25 days & $81.1 \pm 5.1$ & NR & NR \\
\hline 3.0 & 1.0 & Greenish nodular callus found after 25 days & $55.6 \pm 5.1$ & NR & NR \\
\hline 3.0 & 1.5 & Yellowish compact callus found after 25 days & $54.4 \pm 6.9$ & NR & NR \\
\hline
\end{tabular}

* $\mathrm{AM}=$ Arithmetic Mean; $\mathrm{SD}=$ Standard Deviation; $\mathrm{NR}=$ No Response.

Table 5. The effects of BA, KIN and their combinations on callus of Citrus sinensis (L.) Osbeck for shoot regeneration, cultured on MSSIM.

\begin{tabular}{|c|c|c|c|c|c|}
\hline \multicolumn{2}{|c|}{ MS + Hormone $(\mathrm{mg} / \mathrm{l})$} & \multirow[b]{2}{*}{ Observations } & \multirow{2}{*}{$\begin{array}{l}\text { Regeneration } \\
(\mathrm{AM}) \pm \mathrm{SD} \%\end{array}$} & \multicolumn{2}{|c|}{ Organogenesis } \\
\hline BA & KIN & & & $\begin{array}{l}\text { Number of shoot } \\
(A M) \pm S D\end{array}$ & $\begin{array}{c}\text { Root } \\
(\mathrm{AM}) \pm \mathrm{SD}\end{array}$ \\
\hline 0.0 & 0.0 & No shoot formed after 50 days & $0.0 \pm 0.0$ & $0.0 \pm 0.0$ & NR \\
\hline 2.0 & 0.0 & Multiple shoots formed after 45 days & $66.7 \pm 4.6$ & $2.5 \pm 2.0$ & NR \\
\hline 3.0 & 0.0 & Multiple shoots formed after 40 days & $89.3 \pm 2.3$ & $3.5 \pm 1.7$ & NR \\
\hline 4.0 & 0.0 & Multiple shoots formed after 50 days & $69.3 \pm 4.6$ & $3.7 \pm 2.0$ & NR \\
\hline 2.0 & 1.0 & Multiple shoots formed after 40 days & $61.3 \pm 10.1$ & $4.6 \pm 1.6$ & NR \\
\hline 2.5 & 1.5 & Multiple shoots formed after 45 days & $61.3 \pm 2.3$ & $4.4 \pm 1.9$ & NR \\
\hline 3.0 & 1.0 & Multiple shoots formed after 40 days & $72.0 \pm 4.0$ & $3.5 \pm 2.3$ & NR \\
\hline 3.0 & 2.0 & Multiple shoots formed after 45 days & $61.3 \pm 8.3$ & $4.3 \pm 2.1$ & NR \\
\hline
\end{tabular}

* $\mathrm{AM}=$ Arithmetic Mean; $\mathrm{SD}=$ Standard Deviation; $\mathrm{NR}=$ No Response. 
Table 6. The effects of BA, KIN and their combinations on callus of Citrus sinensis (L.) Osbeck for shoot regeneration, cultured on N6SIM.

\begin{tabular}{|c|c|c|c|c|c|}
\hline \multicolumn{2}{|c|}{$\mathrm{N} 6$ + Hormone $(\mathrm{mg} / \mathrm{l})$} & \multirow[b]{2}{*}{ Observations } & \multirow{2}{*}{$\begin{array}{l}\text { Regeneration } \\
(A M) \pm S D \%\end{array}$} & \multicolumn{2}{|c|}{ Organogenesis } \\
\hline BA & KIN & & & $\begin{array}{l}\text { Number of shoot } \\
(A M) \pm S D\end{array}$ & $\begin{array}{c}\text { Root } \\
(\mathrm{AM}) \pm \mathrm{SD}\end{array}$ \\
\hline 0.0 & 0.0 & No shoot formed after 50 days & $0.0 \pm 0.0$ & $0.0 \pm 0.0$ & NR \\
\hline 2.0 & 0.0 & Multiple shoots formed after 45 days & $42.7 \pm 4.6$ & $4.4 \pm 1.8$ & NR \\
\hline 3.0 & 0.0 & Multiple shoots formed after 35 days & $85.3 \pm 6.1$ & $3.5 \pm 1.8$ & NR \\
\hline 4.0 & 0.0 & Multiple shoots formed after 45 days & $73.3 \pm 4.6$ & $3.6 \pm 2.0$ & NR \\
\hline 2.0 & 1.0 & Multiple shoots formed after 35 days & $61.3 \pm 2.3$ & $4.8 \pm 1.6$ & NR \\
\hline 2.5 & 1.5 & Multiple shoots formed after 45 days & $65.3 \pm 4.6$ & $4.2 \pm 1.9$ & NR \\
\hline 3.0 & 1.0 & Multiple shoots formed after 40 days & $76.0 \pm 6.9$ & $3.7 \pm 2.3$ & NR \\
\hline 3.0 & 2.0 & Multiple shoots formed after 30 days & $70.7 \pm 6.1$ & $4.1 \pm 2.1$ & NR \\
\hline
\end{tabular}

* $\mathrm{AM}=$ Arithmetic Mean; $\mathrm{SD}=$ Standard Deviation; $\mathrm{NR}=$ No Response.

Table 7. The effects of BA, KIN and their combinations on callus of Citrus sinensis (L.) Osbeck for shoot regeneration, cultured on B5SIM.

\begin{tabular}{|c|c|c|c|c|c|}
\hline \multicolumn{2}{|c|}{ B5 + Hormone $(\mathrm{mg} / \mathrm{l})$} & \multirow[b]{2}{*}{ Observations } & \multirow{2}{*}{$\begin{array}{l}\text { Regeneration } \\
(\mathrm{AM}) \pm \mathrm{SD} \%\end{array}$} & \multicolumn{2}{|c|}{ Organogenesis } \\
\hline BA & KIN & & & $\begin{array}{l}\text { Number of shoot } \\
(A M) \pm S D\end{array}$ & $\operatorname{Root}(\mathrm{AM}) \pm \mathrm{SD}$ \\
\hline 0.0 & 0.0 & No shoot formed after 40 days & $0.0 \pm 0.0$ & $0.0 \pm 0.0$ & NR \\
\hline 2.0 & 0.0 & Multiple shoots formed after 45 days & $46.7 \pm 4.6$ & $4.2 \pm 1.9$ & NR \\
\hline 3.0 & 0.0 & Multiple shoots formed after 32 days & $77.3 \pm 2.3$ & $3.7 \pm 1.8$ & NR \\
\hline 4.0 & 0.0 & Multiple shoots formed after 45 days & $69.3 \pm 6.1$ & $3.7 \pm 1.5$ & NR \\
\hline 2.0 & 1.0 & Multiple shoots formed after35 days & $50.7 \pm 2.3$ & $4.0 \pm 1.7$ & NR \\
\hline 2.5 & 1.5 & Multiple shoots formed after 40 days & $68.0 \pm 4.0$ & $4.1 \pm 1.5$ & NR \\
\hline 3.0 & 1.0 & Multiple shoots formed after 40 days & $73.3 \pm 4.6$ & $3.4 \pm 2.0$ & NR \\
\hline 3.0 & 2.0 & Multiple shoots formed after 45 days & $62.7 \pm 10.1$ & $4.3 \pm 2.3$ & NR \\
\hline
\end{tabular}

* $\mathrm{AM}=$ Arithmetic Mean; $\mathrm{SD}=$ Standard Deviation; $\mathrm{NR}=$ No Response.

Table 8. Root induction from shoots of Citrus sinensis (L.) Osbeck cultured on MSRtM, N6RtM and B5RtM with supplementation of $5 \mathrm{mg} / \mathrm{l} \mathrm{NAA}$.

\begin{tabular}{|c|c|c|c|c|}
\hline Media & Observations & $\begin{array}{l}\text { Rooting Frequency } \\
(\mathrm{AM}) \pm \mathrm{SD}\end{array}$ & $\begin{array}{l}\text { Root number } \\
(A M) \pm S D\end{array}$ & $\begin{array}{c}\text { Root length } \\
(\mathrm{AM}) \pm \mathrm{SD}(\mathrm{cm})\end{array}$ \\
\hline MSRtM & Multiple roots observed after 30 - 40 days & $64.4 \pm 6.9$ & $4.6 \pm 1.8$ & $2.9 \pm 0.9$ \\
\hline N6RtM & Multiple roots observed after 25 - 40 days & $74.4 \pm 5.1$ & $4.9 \pm 1.9$ & $2.2 \pm 0.7$ \\
\hline B5TtM & Multiple roots observed after 30 - 50 days & $53.3 \pm 11.5$ & $4.4 \pm 2.6$ & $2.7 \pm 0.6$ \\
\hline
\end{tabular}

* $\mathrm{AM}=$ Arithmetic Mean; $\mathrm{SD}=$ Standard Deviation. 


\section{Results and Discussions}

The present research was carried out to develop a reproducible regeneration protocol for Citrus sinensis (L.) Osbeck from mature seed-derived calli. According to previous reports, supplementation of 2,4-D in the medium induce a high percentage of callus in Citrus [16]. High-frequency callus initiation has also been achieved using NAA [17] and also the combination of 2,4 D and NAA [18]. In this study, mature Citrus seeds were used as explants and were cultured using MS, N6 and B5 media containing different concentrations and combinations of plant growth regulators. MS medium is the most used basal medium for the in-vitro callus induction [19]. In this research, we investigated the effects of MS, N6 and B5 basal media as well as the effects of plant growth regulators in in-vitro culture of Citrus sinensis (L.) Osbeck [20]. Different callus induction frequencies were observed in different treatments. Callus induction frequency on MS medium was found higher than N6 and B5 media. Callus induction frequencies changed with the variation of hormonal concentrations in the same medium. Maximum frequency was $86.7 \% \pm 3.4 \%$ in MSCIM media when supplemented with $3 \mathrm{mg} / \mathrm{l} 2,4-\mathrm{D}, 83.3 \% \pm 8.8 \%$ in N6CIM media when supplemented with $2.5 \mathrm{mg} / \mathrm{l} 2,4-\mathrm{D}$ and $82.2 \% \pm 1.9 \%$ in B5CIM medium when supplemented with $3.5 \mathrm{mg} / 1$ 2,4-D. Nearly similar results were found in previous reports [16] [21] [22]. Supplementation of NAA also showed varied results in callus induction but the frequency of callus induction was relatively lower than 2,4-D. Highest callus induction frequency was observed in MSCIM and N6CIM media with supplementation of $4.0 \mathrm{mg} / \mathrm{l} \mathrm{NAA}$ and relatively lower frequency was found in B5CIM media at the same concentration. Combination of 2,4-D and NAA showed relatively similar results to that of 2,4-D supplemented media [23]. Highest callus induction frequency of $80.0 \% \pm 3.3 \%$ was observed on MSCIM medium when a combination of $2.0 \mathrm{mg} / \mathrm{l} 2,4-\mathrm{D}$ and $1.5 \mathrm{mg} / \mathrm{l} \mathrm{NAA}$ was used. N6CIM media showed $77.8 \% \pm 6.9 \%$ callus induction frequency at $2.0 \mathrm{mg} / \mathrm{l} 2$, 4-D and $1.5 \mathrm{mg} / \mathrm{l} \mathrm{NAA}$ supplementation. But B5CIM showed relatively higher callus induction frequency of $81.1 \% \pm 5.1 \%$ at $3.0 \mathrm{mg} / 12,4$-D and $0.5 \mathrm{mg} / \mathrm{l} \mathrm{NAA}$ supplementation (Figure 1). The color of the calli was varied with treatments and found greenish, yellowish, whitish and light greenish calli. Two types, both embryogenic and non-embryogenic calli were found in this experiment (Figure 2). For induction of shoot, only embryogenic calli were used as explants. The selected calli were inoculated on MSSIM, N6SIM, and B5SIM media containing different concentrations of BA and combinations of BA and Kinetin (KIN) (Table 5-7). After three weeks of inoculation, shoots started to form. After four weeks, the shoots grew $1-3 \mathrm{~cm}$ long in length (Figure 3). Different treatments resulted in various shoot induction frequencies. MSSIM medium supplemented with $3 \mathrm{mg} / \mathrm{l}$ BA showed maximum shoot induction frequency which was $89.3 \%$ $\pm 2.3 \%$. The findings of shoot induction using BA and KIN support the previous reports [21] [23] [24]. N6SIM and B5SIM also showed maximum frequency at $3.0 \mathrm{mg} / \mathrm{l} \mathrm{BA}$ supplementation $85.3 \% \pm 6.1 \%$ and $77.3 \% \pm 2.3 \%$ respectively. 
Combinations of BA and KIN showed relatively lower frequencies (Figure 4). Earlier reports suggest that MS media with supplementation of different growth regulators like BA, NAA, and IBA promotes efficient root formation [25] [26] [27] [28]. The regenerated shoots were transferred to MSRtM, N6RtM and B5RtM medium with supplementation of $5 \mathrm{mg} / \mathrm{l} \mathrm{NAA}$. After 18 days of inoculation, root induction was started to begin and the three media showed different root induction frequencies (Figure 5). Maximum root induction frequency was observed in N6RtM medium which was $74.4 \% \pm 5.1 \%$ where MSRtM and B5RtM medium showed relatively lower root induction frequencies which were $64.4 \% \% \pm 6.9 \%$ and $53.3 \% \pm 11.5 \%$ respectively (Table 8 ). Large numbers of embryonic calli derived plantlets were successfully acclimatized to ex-vitro conditions. The roots of plantlets were washed and placed in pots containing garden soil mixed with biofertilizer in $1: 1$ ratio in a culture room at $25^{\circ} \mathrm{C} \pm 2^{\circ} \mathrm{C}$ for 10 days. The survival rate of plants was $60 \%$ from all media derived plantlets. After 15 days, plantlets were exposed to natural conditions. After 40 days, plants showed a significant growth performance. The plants regenerated on MSRtM medium showed significantly high survivability in the natural conditions.

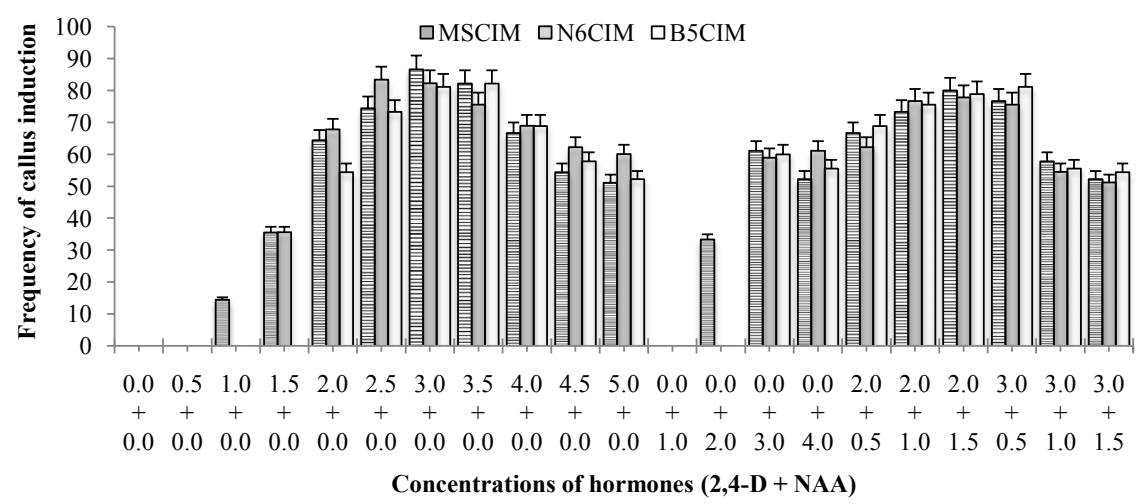

Figure 1. Effect of PGRs (2,4-D, NAA and combinations) and different basal media in callus induction of Citrus sinensis (L.) Osbeck.

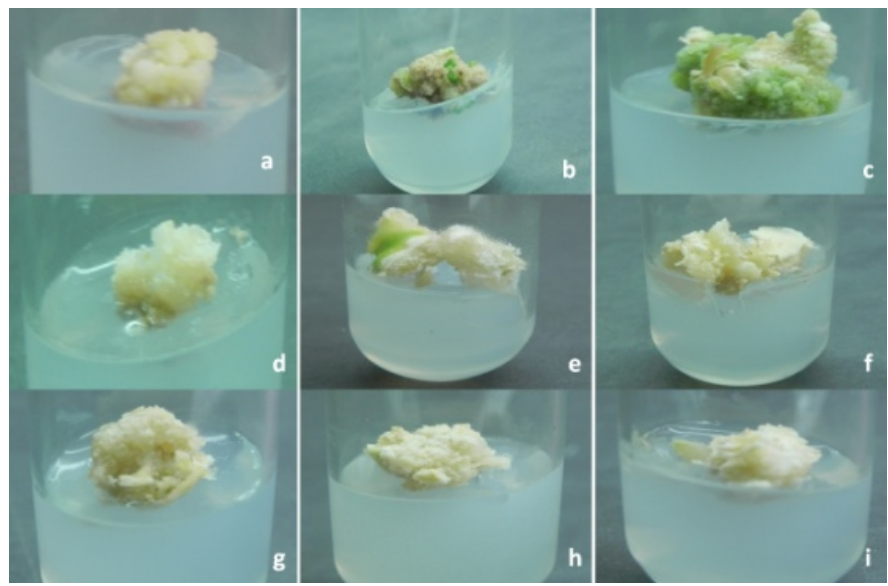

Figure 2. Effect of 2, 4-D on callus induction of Citrus sinensis (L.) Osbeck in different basal medium; (Here, a-c: MSCIM; d-f: N6CIM and g-i: B5CIM). 


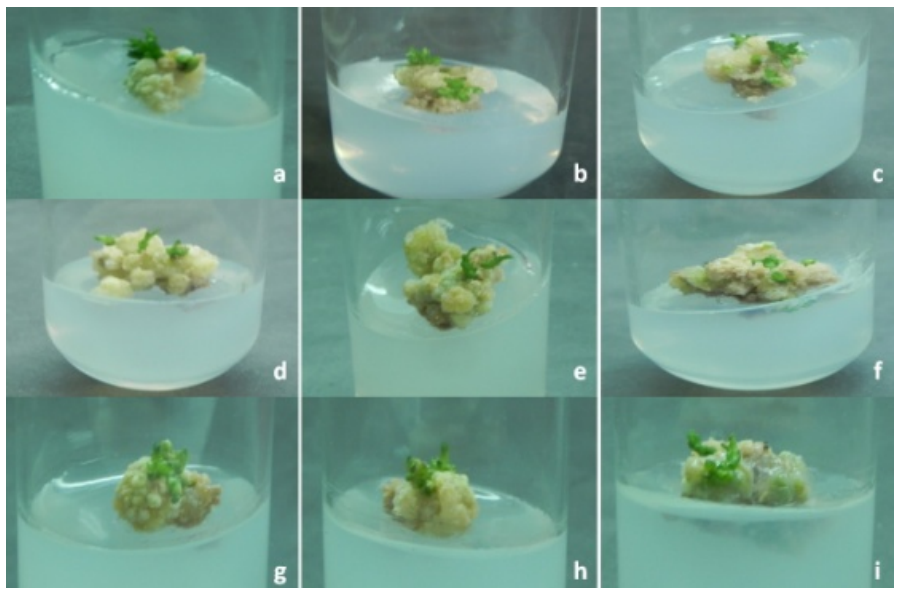

Figure 3. Shoot regeneration from callus of Citrus sinensis (L.) Osbeck using BA and KIN on different basal medium; (Here, a-c: MSSIM; d-f: N6SIM and g-i: B5SIM).

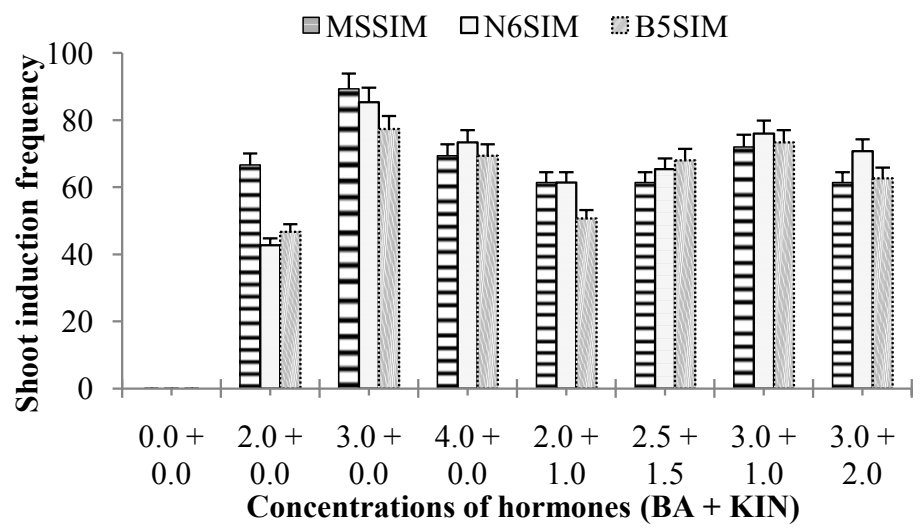

Figure 4. Effect of PGRs (BA, combination of BA and KIN) and different basal media in shoot induction of Citrus sinensis (L.) Osbeck.

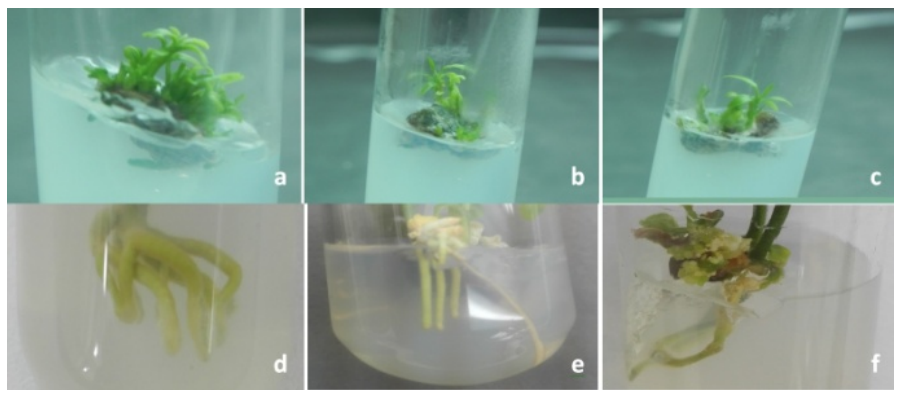

Figure 5. Root induction from shoot of Citrus sinensis (L.) Osbeck with supplementation of $5 \mathrm{mg} / \mathrm{l} \mathrm{NAA}$ on different basal medium; (Here, a, d: MSRtM; b, e: N6RtM and c, f: B5RtM).

\section{Conclusion}

This study of in-vitro callus induction and regeneration of Citrus sinensis (L.) Osbeck was carried out to investigate the appropriate hormones with their optimum concentration and suitable media for callus induction, shoot generation and root formation. In-vitro callus inductions were investigated using three bas- 
al media supplemented with 2,4-D, NAA alone and their combinations. Supplementation of $3 \mathrm{mg} / \mathrm{l}$ 2,4-D in all three media (MSCIM, N6SIM, and B5SIM) showed maximum callus induction frequencies and MSCIM media showed higher frequency compared to N6SIM and B5SIM. Shoot induction from embryogenic calli was investigated on MSSIM, N6SIM and B5SIM media with supplementation of different concentrations of BA, KIN alone and their combinations. Maximum shoot inductions were observed at $3 \mathrm{mg} / \mathrm{l} \mathrm{BA}$ supplementation but the combination of the two hormones showed relatively lower shoot induction frequencies. On the other hand, maximum shoots induction frequency was observed on MSSIM medium compared to N6SIM and B5SIM. Root induction was investigated on MSRtM, N6RtM and B5RtM media with supplementations of $5 \mathrm{mg} / \mathrm{l}$ NAA. Maximum rooting frequency was observed on N6RtM but maximum survivability was observed from the plantlets which were regenerated on MSRtM. This study demonstrated that the $3 \mathrm{mg} / \mathrm{l} 2,4-\mathrm{D}$ supplementation on MSCIM medium has the maximum potential to incite callus formation from matured Citrus sinensis (L.) Osbeck seeds. $3 \mathrm{mg} / \mathrm{l}$ BA supplementation on MSSIM medium has the maximum potential to prompt shoots. MSRtM and N6RtM medium with supplementations of $5 \mathrm{mg} / \mathrm{l}$ NAA has the potential to promote roots.

\section{Acknowledgements}

This work was performed in Plant Genetic Engineering and Biotechnology Laboratory at Department of Genetic Engineering and Biotechnology, Shahjalal University of Science and Technology, Sylhet-3114, Bangladesh. This work was financially supported by the Ministry of Science and Technology, the People's Republic of Bangladesh.

\section{Conflicts of Interest}

The authors declare no conflicts of interest regarding the publication of this paper.

\section{References}

[1] Xu, Q., Chen, L.-L., Ruan, X.A., Chen, D.J., Zhu, A.D., et al. (2013) The Draft Genome of Sweet Orange (Citrus sinensis). Nature Genetics, 45, 59-66. https://doi.org/10.1038/ng.2472

[2] Roose, M.L. and Close, T.J. (2008) Genomics of Citrus, a Major Fruit Crop of Tropical and Subtropical Regions. In: Moore, P.H. and Ming, R., Eds., Genomics of Tropical Crop Plants. Springer, Berlin, 187-202. https://doi.org/10.1007/978-0-387-71219-2_8

[3] Bausher, M.G., Singh, N.D., Lee, S.-B., Jansen, R.K. and Daniell, H. (2006) The Complete Chloroplast Genome Sequence of Citrus sinensis (L.) Osbeck var "Ridge Pineapple": Organization and Phylogenetic Relationships to Other Angiosperms. BMC Plant Biology, 6, 21. https://doi.org/10.1186/1471-2229-6-21

[4] Grosser, J., et al. (2005) Applications of Somatic Hybridization and Cybridization in Scion and Rootstock Improvement, with Focus on Citrus. In International Sympo- 
sium on Biotechnology of Temperate Fruit Crops and Tropical Species, 738.

[5] Rezadost, M.H., et al. (2013) In Vitro Regeneration of Sour Orange (Citrus aurantium L.) via Direct Organogenesis. Plant Knowledge Journal, 2, 150.

[6] García-Luis, A., Molina, R.V., Varona, V., Castelló, S. and Guardiola, J.L. (2006) The Influence of Explant Orientation and Contact with the Medium on the Pathway of Shoot Regeneration in Vitro in Epicotyl Cuttings of Troyer citrange. Plant Cell, Tissue and Organ Culture, 85, 137-144. https://doi.org/10.1007/s11240-005-9060-4

[7] Prodhan, S.H., et al. (2016) Development of an Efficient in Vitro Regeneration System for Endangered Wild Orange Citrus chrysocarpa L. International Journal of Sciences. Basic and Applied Research (IJSBAR), 30, 187-196.

[8] Cervera, M., Juarez, J., Navarro, A., Pina, J.A., Duran-Vila, N., Navarro, L., et al. (1998) Genetic Transformation and Regeneration of Mature Tissues of Woody Fruit Plants Bypassing the Juvenile Stage. Transgenic Research, 7, 51-59. https://doi.org/10.1023/A:1008855922283

[9] Bond, J. and Roose, M. (1998) Agrobacterium-Mediated Transformation of the Commercially Important Citrus Cultivar Washington Navel Orange. Plant Cell Reports, 18, 229-234. https://doi.org/10.1007/s002990050562

[10] Curtis, I.S. and Mirkov, T.E. (2012) Influence of Surfactants on Growth and Regeneration from Mature Internodal Stem Segments of Sweet Orange (Citrus sinensis) cv. Hamlin. Plant Cell, Tissue and Organ Culture (PCTOC), 108, 345-352. https://doi.org/10.1007/s11240-011-0037-1

[11] Hasan, M.R., et al. (2016) Efficient Regeneration System for the Improvement of Kinnow Mandarin (Citrus reticulata Blanco). Journal of Biology, Agriculture and Healthcare, 6, 39-47.

[12] Almeida, W.A.B.D., de Assis Alves Mourão Filho, F., Mendes, B.M.J. and Rodriguez, A.P.M. (2002) In Vitro Organogenesis Optimization and Plantlet Regeneration in Citrus sinensis and C. limonia. Scientia Agricola, 59, 35-40. https://doi.org/10.1590/S0103-90162002000100004

[13] Azim, F., Rahman, M., Prodhan, S., Sikdar, S., Zobayer, N. and Ashrafuzzaman, M. (2011) Development of Efficient Callus Initiation of Malta (Citrus sinensis) through Tissue Culture. International Journal of Agricultural Research, Innovation and Technology, 1, 64-68. https://doi.org/10.3329/ijarit.v1i1-2.13935

[14] Hasan, M.R., et al. (2016) Efficient Callus Initiation and Plantlet Regeneration of Citrus japonica Margarita. IOSR Journal of Pharmacy and Biological Sciences (IOSR-JPBS), 11, 72-78.

[15] Yaacob, J.S., et al. (2014) Optimization of Culture Conditions (Sucrose, pH, and Photoperiod) for in Vitro Regeneration and Early Detection of Somaclonal Variation in Ginger Lime (Citrus assamensis). The Scientific World Journal, 2014, Article No. 262710.

[16] Kiong, A.L.P., Wan, L.S., Hussein, S. and Ibrahim, R. (2008) Induction of Somatic Embryos from Different Explants of Citrus sinensis. Journal of Plant Sciences, 3, 18-32. https://doi.org/10.3923/jps.2008.18.32

[17] Mukhtar, R., et al. (2005) In Vitro Regeneration and Somatic Embryogenesis in (Citrus aurantifolia and Citrus sinensis). International Journal of Agriculture and Biology, 7, 414-416.

[18] Pandey, A. and Tamta, S. (2016) Efficient Micropropagation of Citrus sinensis (L.) Osbeck from Cotyledonary Explants Suitable for the Development of Commercial Variety. African Journal of Biotechnology, 15, 1806-1812. 
https://doi.org/10.5897/AJB2015.14986

[19] Germana, M.A., Micheli, M., Chiancone, B., Macaluso, L. and Standardi, A. (2011) Organogenesis and Encapsulation of in Vitro-Derived Propagules of Carrizo Citrange [Citrus sinensis (L.) Osb. $\times$ Poncirius trifoliata (L.) Raf]. Plant Cell, Tissue and Organ Culture, 106, 299-307. https://doi.org/10.1007/s11240-011-9921-y

[20] Esmaeilnia, E. and Dehestani, A. (2015) In Vitro Plant Regeneration from Mature Tissues of Thomson Navel Sweet Orange (Citrus sinensis L. Osbeck.). Biharean Biologist, 9, 9-14.

[21] Badrelden, A. (2017) Establishment of in Direct Propagation of Mandarin (Citrus reticulata L.) Using Tissue Culture. Egyptian Journal of Botany, 57, 405-416. https://doi.org/10.21608/ejbo.2017.799.1050

[22] Ramdan, R., et al. (2014) Influence of Growth Regulators on Callus Induction from Embryos of Five Citrus Rootstocks. Journal of Applied Biosciences, 73, 5959-5965.

[23] Singh, B., Virk, G.S. and Nagpal, A.K. (2011) An Efficient Plant Regeneration Protocol from Callus Cultures of Citrus jambhiri Lush. Physiology and Molecular Biology of Plants, 17, 161-169. https://doi.org/10.1007/s12298-011-0055-9

[24] Kasprzyk-Pawelec, A., Pietrusiewicz, J. and Szczuka, E. (2015) In Vitro Regeneration Induced in Leaf Explants of Citrus limon L. Burm cv. "Primofiore". Acta Scientiarum Polonorum Hortorum Cultus, 14, 143-153.

[25] Haripyaree, A., et al. (2011) In Vitro Propagation of Citrus megaloxycarpa. Environmental and Experimental Biology, 9, 129-132.

[26] Ali, S., Mannan, A., El Oirdi, M., Waheed, A. and Mirza, B. (2012) Agrobacterium-Mediated Transformation of Rough Lemon (Citrus jambhiri Lush) with Yeast HAL2 Gene. BMC Research Notes, 5, 285. https://doi.org/10.1186/1756-0500-5-285

[27] Peña, L., Cervera, M., Juárez, J., Navarro, A., Pina, J.A., Durán-Vila, N., et al. (1995) Agrobacterium-Mediated Transformation of Sweet Orange and Regeneration of Transgenic Plants. Plant Cell Reports, 14, 616-619. https://doi.org/10.1007/BF00232724

[28] Usman, M., Sana, M. and Fatima, B. (2006) In Vitro Multiple Shoot Induction from Nodal Explants of Citrus Cultivars. Journal of Central European Agriculture, 6 , 435-442. 